\title{
Detection and characterization of prednisolone metabolites in human urine by LC-MS/MS
}

Xavier Mataboscha, Oscar J. Pozo , Clara Pérez-Mañáb,c, Esther Papaseit b,c , Jordi Segura $^{\mathrm{a}, \mathrm{d}}$, Rosa Ventura ${ }^{\mathrm{a}, \mathrm{d}^{*}}$

a Bioanalysis Research Group, IMIM, Institut Hospital del Mar d’Investigacions Mèdiques , Doctor Aiguader 88, 08003 Barcelona, Spain

b Human Pharmacology and Clinical Neurosciences Research Group, IMIM, Institut Hospital del Mar d’Investigacions Mèdiques, Doctor Aiguader 88, 08003 Barcelona, Spain

${ }^{\mathrm{c}}$ Department of Pharmacology, Therapeutics and Toxicology, Universitat Autònoma de Barcelona, Bellaterra (Cerdanyola del Vallès), 08193 Barcelona, Spain

${ }^{\mathrm{d}}$ Department of Experimental and Health Sciences, Universitat Pompeu Fabra, Doctor Aiguader 88, 08003 Barcelona, Spain

Running Head: Prednisolone metabolites in human urine

Corresponding author: Rosa Ventura

Phone: 0034-933160471

Fax: 0034-933160499

E-mail: rventura@imim.es 


\section{ABSTRACT}

Glucocorticosteroids are prohibited in sports when using by systemic administrations (e.g. oral), whereas they are allowed using other administration ways. Strategies to discriminate between administrations routes have to be developed by doping control laboratories. For this reason, the metabolism of prednisolone (PRED) was studied using liquid chromatography coupled to tandem mass spectrometry (LC-MS/MS). A single oral (10 mg) dose of PRED was administrated to two healthy male volunteers. Urine samples were collected up to 6 days after administration. Samples were hydrolyzed with $\beta$-glucuronidase and subjected to liquid-liquid extraction with ethyl acetate in alkaline conditions. The extracts were analysed by liquid chromatography coupled to tandem mass spectrometry. Precursor ion scan methods $(\mathrm{m} / \mathrm{z} 77,91,105,121,147,171)$ in positive ionization and neutral loss scan methods (76 and $94 \mathrm{Da}$ ) in negative ionization mode were applied for the open detection of PRED metabolites. Using these methods, PRED parent compound plus 20 metabolites were detected. PRED and eleven metabolites were characterized by

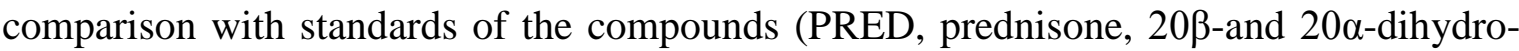

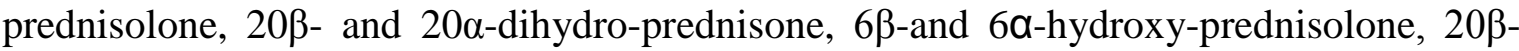
and $20 \alpha$ isomers of $6 \beta, 11 \beta, 17 \alpha, 20,21$-pentahydroxypregnan-1,4-diene-3-one, $6 \alpha, 11 \beta, 17 \alpha, 20 \beta, 21$-pentahydroxypregnan-1,4-diene-3-one and $\Delta^{6}$-PRED). Using mass spectrometric data, feasible structures were proposed for seven of the remaining nine detected metabolites, including several 6-hydroxy-metabolites. Eleven of the characterized metabolites have not been previously described. Maximum excretion rates for PRED metabolites were achieved in first 24h, however, most of the metabolites were still detectable in the last collected samples (day 6).

Keywords: prednisolone, metabolism, mass spectrometry, doping analysis, corticosteroid 



\section{INTRODUCTION}

Corticosteroid use in sport competition is prohibited by the World Anti-Doping Agency (WADA) when administered by oral, intravenous, intramuscular or rectal routes, and they are allowed using other administration routes. ${ }^{[1]}$ In an attempt to discriminate between permitted and forbidden administrations, WADA established a reporting level of $30 \mathrm{ng} / \mathrm{mL}$ for glucocorticosteroids and their metabolites. ${ }^{[2]}$ However, it is known that this reporting level is not suitable for all corticosteroids for many reasons like wide range of therapeutic doses, different administration routes or different excretion rates in urine, among others. Differences in the excretion of metabolites in urine depending on the route of administration have been described for some doping agents, including corticosteroids. ${ }^{[3-10]}$ A detailed study of budesonide metabolism made possible the selection of $6 \beta$-hydroxybudesonide as the best marker to distinguish allowed inhaled from forbidden oral administrations of budesonide. ${ }^{[5,7]}$ Such metabolite has been recently adopted in WADA regulations as a marker to distinguish allowed and forbidden use of budesonide. ${ }^{[2]}$ For methylprednisolone, a comparison of topical and oral administrations showed that the use of some metabolites would improve the distinction between these administration routes compared to targeting on the parent drug. ${ }^{[6,8]}$ For triamcinolone acetonide, the current reporting level applied to the parent compound is not suitable to detect forbidden intramuscular misuse. ${ }^{[9,10]}$

Prednisolone (11ß,17 $\alpha, 21$-trihydroxypregnan-1,4-diene-3,20-dione, PRED, Fig. 1), and prednisone (17 $\alpha, 21$-dihydroxypregnan-1,4-diene-3,11,20-trione) were the first synthetic glucocorticosteroids ${ }^{[11]}$ and have been used as drugs since 1950 s. $^{[12]}$ They are used as antiinflammatory and immunosuppressive agents for a wide range of disease states. Prednisone is both a prodrug and a metabolite of the active drug PRED. ${ }^{[13]}$ Some metabolites were detected in late 50s using paper chromatography (Florisil) ${ }^{[14]}$ and the number of identified 
and characterized metabolites has increased since the use of chromatography coupled to mass spectrometry. ${ }^{[15-18]}$ The previously reported metabolites of PRED are: prednisone, ${ }^{[15-}$

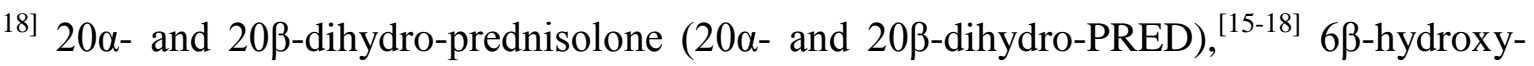
prednisolone $(6 \beta O H-P R E D),{ }^{[15,16,18]} 20 \alpha$ - and $20 \beta$-dihydro-prednisone, ${ }^{[17,18]}$ several isomers of 4,5-dihydroprednisolone ${ }^{[15,18]}$ and 4,5-dihydroprednisone, ${ }^{[15,18]}$ 3,4,5tetrahydroprednisolone, ${ }^{[16]} 3,4,5$-tetrahydroprednisone ${ }^{[16,17]}$ and the $20 \alpha$ - and $20 \beta$ isomers of a 3,4,5,20-hexahydroprednisone. ${ }^{[16]}$

Liquid chromatography coupled to tandem mass spectrometry (LC-MS/MS) has demonstrated to be a useful tool for the identification of new metabolites of compounds with steroid structure, including glucocorticosteroids. ${ }^{[4-6,19-22]}$ The use of this technology is especially important for glucocorticosteroids due to the difficulties to obtain adequate derivatives of polyoxygenated metabolites to make them amenable to gas chromatographymass spectrometry analysis. ${ }^{[23]}$

The goal of the present work was to study PRED metabolism using LC-MS/MS technology to obtain a more extensive picture of its metabolic pathways to be able in a future to evaluate the excretion profiles of the detected PRED metabolites after different routes of administration to find a good marker to discriminate between forbidden and therapeutic administrations of PRED in sports drug testing.

\section{MATERIALS AND METHODS}

\subsection{Chemicals and reagents}

Prednisolone (PRED), cortisone, analytical grade di-sodium hydrogen phosphate, sodium hydrogen phosphate, ethyl acetate, ammonium chloride, acetonitrile and methanol (LC gradient grade), formic acid (LC-MS grade) were obtained from Merck (Darmstadt, Germany). Prednisone (M-I), cortisol, ammonium formate, and sodium borohydride were 
obtained from Sigma (St. Louis, MO, US). 6 $\beta$-hydroxy-prednisolone (6ßOH-PRED, MVI), 6,7-dehydroprednisolone ( $\Delta^{6}$-PRED, M-XI), 16 $\alpha$-hydroxy-prednisolone $(16 \alpha \mathrm{OH}$ PRED), prednisolone-2,4,6,6,9,11,12,12- $\mathrm{d}_{8}$ (PRED- $\mathrm{d}_{8}$ ) and 6 3 -hydroxy-dexamethasone (6ßOH-dexamethasone) were purchased from Toronto Research Chemicals (Toronto,

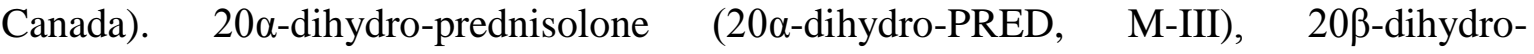

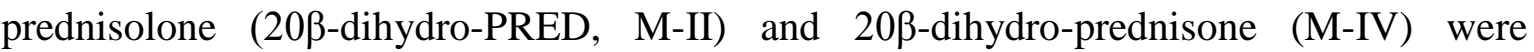
obtained from Steraloids (Newport, RI, USA). The $\beta$-glucuronidase preparation (type $E$. Coli K12) was purchased from Roche Diagnostics GmbH (Mannheim, Germany). The Sep-Pak® Vac RC (500mg) C18 cartridges were purchased from Waters (Milford, MA, USA). A Milli-Q purification system was used (Millipore Ibérica, Spain).

\subsection{LC-MS/MS instrumentation}

LC-MS/MS analyses were carried out using a triple quadrupole mass spectrometer (Xevo TQ MS) provided with an orthogonal Z-spray-electrospray interface (ESI) (Waters Associates, Milford, MA, USA) interfaced to an ultraperformance liquid chromatographic (UHPLC) system, Acquity (Waters Associates) for the chromatographic separation. Nebulising gas, cone gas as well as desolvation gas was nitrogen. The desolvation gas flow was set to approximately $1200 \mathrm{~L} / \mathrm{h}$ and the cone gas flow to $50 \mathrm{~L} / \mathrm{h}$. The nitrogen desolvation temperature was set to $450^{\circ} \mathrm{C}$ and the source temperature to $120^{\circ} \mathrm{C}$. Argon was used as collision gas. Capillary voltages were set at 3.5 and $3.0 \mathrm{kV}$ in positive and negative ionization mode, respectively.

Open scan methods applied in positive or negative ionization modes to detect PRED metabolites are described in Table 1 . A window from $\mathrm{m} / \mathrm{z} 300$ to 600 was monitored for all methods. 
Collision induced dissociation (CID) of the precursor ions, $[\mathrm{M}+\mathrm{H}]^{+}$or $[\mathrm{M}+\mathrm{HCOO}]^{-}$, were also performed in positive and negative ionization modes, respectively. In positive mode, the cone voltage (CV) was set to $10 \mathrm{~V}$ for all metabolites and two different collision energies (CE) were used (10 and 30eV). In negative mode, the CV was set to $15 \mathrm{~V}$ and the CE to 10 or $15 \mathrm{eV}$.

The LC separation was performed using an Acquity BEH $\mathrm{C}_{18}$ column (100 x $2.1 \mathrm{~mm}$ i.d., $1.7 \mu \mathrm{m}$ particle size) (Waters Associates), at a flow rate of $400 \mu \mathrm{L} / \mathrm{min}$ and at $45^{\circ} \mathrm{C}$. Water $1 \mathrm{mM}$ of ammonium formate (solvent A) and acetonitrile (solvent B) both with formic acid (0.01\%) were selected as mobile phase solvents. The percentage of organic solvent was linearly changed as follows: 0 min., $8 \%$; 8 min., 15\% ; 9 min., 20\% ; 14 min., 23\% ; 15 min., 90\% ; 16 min., 90\% ; 16.1 min, 8\% ; 18.5 min., 8\%.

\subsection{Sample preparation}

Internal standard (ISTD) solution (40 ng of PRED-d 8 and 40 ng of $6 \beta \mathrm{OH}$-dexamethasone) was added to $2 \mathrm{~mL}$ of urine, followed by the addition of $0.5 \mathrm{~mL}$ of phosphate buffer $1 \mathrm{M}$ pH 7. Then, $\beta$-glucuronidase from E. coli was added $(30 \mu \mathrm{L})$ and hydrolysis was carried out for $1 \mathrm{~h}$ at $55^{\circ} \mathrm{C}$. The buffered solution was alkalinized with $150 \mu \mathrm{L}$ of $25 \%$ potassium carbonate solution to $\mathrm{pH}$ 8-9 and the steroids were extracted with $6 \mathrm{~mL}$ of ethyl acetate. After centrifugation (5 minutes at $1400 \mathrm{~g}$ ) the organic layer was transferred into a new tube and evaporated to dryness under nitrogen stream in a water bath at $40^{\circ} \mathrm{C}$. The residue was reconstituted into $100 \mu \mathrm{L}$ of a mixture of water:acetonitrile (75:25, v/v) and $10 \mu \mathrm{L}$ were directly injected into the system.

\subsection{Chemical reduction}


Chemical reduction of the C20 carbonyl group of $6 \beta \mathrm{OH}-\mathrm{PRED}$ was performed. A modified version of a previously described method was applied. ${ }^{[24]}$ Reduction was performed by adding $100 \mathrm{mg}$ of $\mathrm{NaBH}_{4}$ to a solution of $6 \beta \mathrm{OH}-\mathrm{PRED}(10 \mu \mathrm{g})$ in $6 \mathrm{~mL}$ of methanol and $1 \mathrm{~mL}$ of water. After stirring the mixture at room temperature for $5 \mathrm{~h}$, a few drops of acetic acid were added. After evaporating methanol, aqueous solutions were passed through a C18 cartridge, previously conditioned with $2 \mathrm{~mL}$ of methanol and $2 \mathrm{~mL}$ of water. The column was then washed with $2 \mathrm{~mL}$ of water and finally analytes were eluted with $2 \mathrm{~mL}$ of methanol. Methanol was evaporated to dryness, and reaction products were reconstituted into $100 \mu \mathrm{L}$ of a mixture of water:acetonitrile (75:25, v/v) and $10 \mu \mathrm{L}$ were analyzed by LC-MS/MS.

\subsection{Clinical Studies.}

A single oral dose of $10 \mathrm{mg}$ of PRED (prednisolone steaglate, Estilsona oral drops, Sonphar, Spain) was administered to two healthy male Caucasian volunteers (volunteer 1, 27 years old; and volunteer 2, 23 years old). Urine samples were collected baseline and from 0-8h, 8-24h, 24-36h, 36-48h, 48-72h, 72-96h, 96-120h and 120-144h after drug administration and stored at $-20^{\circ} \mathrm{C}$ until analysis.

Ethical approval for the study had been granted by the Ethical Committee of our Institute (Comité Ètic d’Investigació Clínica CEIC-Parc de Salut Mar, Barcelona, Spain) and the Spanish Medicines Agency, (EudraCT protocol number 2010-021237-31). Both subjects participating in the study gave their written informed consent prior inclusion and underwent a general physical examination, routine laboratory test, urinalysis, and a 12-lead electrocardiogram.

\subsection{Calculation of the excretion profile.}


To evaluate the excretion profiles of PRED metabolites after oral administration of the drug, the area ratios of all metabolites to the ISTD were calculated. The ISTDs, $6 \beta \mathrm{OH}-$ dexamethasone and PRED- $\mathrm{d}_{8}$, were used for metabolites eluting in the ranges 0-9 min and 9-15 min, respectively (see Table 3). In order to reduce variability, the area ratios of the analytes were normalized by means of the urinary density. The ratios per sample were corrected to a specific gravity of 1.020 applying the following formula: ${ }^{[25]}$

Corrected Area Ratio ${ }_{1.020}=$ Area ratio $_{\text {measured }}$ X (1.020-1) / (specific gravity sample $_{\text {- }}$ )

\section{RESULTS AND DISCUSSION}

\subsection{Ionization and fragmentation of corticosteroids}

The MS behavior of PRED and related compounds available as reference standards was studied in order to find characteristic ionization and fragmentation behaviors that could help in the characterization of potential metabolites of PRED. All compounds were ionized in positive mode at the conjugated 3-keto function to form the $[\mathrm{M}+\mathrm{H}]^{+}$ion and, in negative mode, they were ionized by formation of the adduct $[\mathrm{M}+\mathrm{HCOO}]^{-}$, as described for other corticosteroids. ${ }^{[4-6,9,26]}$ CID mass spectra of the precursor ions $[\mathrm{M}+\mathrm{H}]^{+}$and $[\mathrm{M}+\mathrm{HCOO}]^{-}$ are summarized in Table 2, and they are shown in Fig. S1 (supporting information).

Compounds showed the typical fragmentation of corticosteroids. ${ }^{[4-6,9]}$ In positive mode, ions at $\mathrm{m} / \mathrm{z}$ values higher than 275 were originated mainly by typical losses of $\mathrm{H}_{2} \mathrm{O}$, and in less extent by losses of $28 \mathrm{Da}$ (typical of a unconjugated ketone group). ${ }^{[4-6,9]}$ For $20 \beta$ dihydro-prednisone, a NL of 30Da had higher abundance than the expected NL of 28 Da. In previous studies with methylprednisolone metabolites, ${ }^{[6]}$ the abundance of the NL of 28Da was 10 times higher for 11-keto-methylprednisolone compared to 20-dehydro-11keto-methylprednisolone. Thus, these results suggest that both ketones in C11 and C20 are needed to detect this fragmentation in moderate intensities. A NL of 60 Da, previously 
observed in C20 reduced metabolites of betamethasone, ${ }^{[26]}$ was also seen in C20 reduced PRED related compounds. This NL was confirmed by MS/MS of in-source fragmented

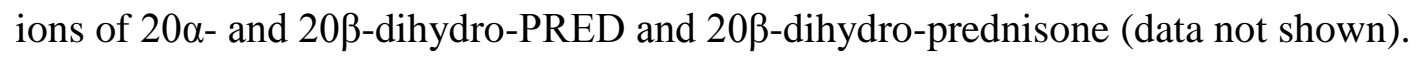

At CE of $30 \mathrm{eV}$, ions at $\mathrm{m} / \mathrm{z} 121,147$ and 171, typical of 1,4-diene-corticosteroids ${ }^{[4-6,9]}$ were detected (Fig. S1). The base peak at $\mathrm{m} / \mathrm{z} 171$ and low abundance of peaks at $\mathrm{m} / \mathrm{z} 121$ and 147 of $\Delta^{6}$-PRED were in agreement with mass spectra of previously described 6,7dehydrometabolites of budesonide ${ }^{[5]}$ and of methylprednisolone. ${ }^{[6]}$

As expected, ${ }^{[9]}$ in negative ionization, the main product ion was obtained after a NL of 76 Da (formic acid and formaldehyde) for 20-keto compounds, and after a NL of 46 Da (formic acid) for 20-dihydro analogs.

\subsection{Detection of PRED metabolites}

Prior to the analysis of excretion study samples a UHPLC gradient was optimized for the separation of all commercially available PRED metabolites. Retention times of endogenous cortisol and cortisone were also evaluated because they have the same molecular mass as potential metabolites of PRED, such as 20-dihydro-PRED or 20dihydro-prednisone. Chromatographic separation of 20ß-dihydro-PRED and cortisol could not be achieved using methanol/water as mobile phase solvents. Complete separation of all isobaric studied standards was achieved using acetonitrile/water as mobile phase solvents. Retention times for PRED metabolites are indicated in Table 3, and those of endogenous cortisol, and cortisone were $13.7 \mathrm{~min}$ and $13.8 \mathrm{~min}$, respectively.

Detection and identification of PRED metabolites were accomplished by analysis by LCMS/MS of a $20 \mathrm{~mL}$ urine extract collected in the range 0 -8h after oral intake. Ten aliquots of $2 \mathrm{~mL}$ of the sample were extracted and combined. Results were compared with those obtained after analysis of the same volume of a pre-administration urine. LC-MS/MS 
methods based on both precursor ion (PI) and neutral loss (NL) scans in positive and negative modes were applied (Table 1). These methods allow for the detection of unknown metabolites of corticosteroids that share common structural features ${ }^{[4]}$ and its usefulness has been proven with several corticosteroids. ${ }^{[4-6,9,26]}$ After combining the information of all PI and NL methods, contrasting the results between post- and pre-administration samples and checking in scan mode the nature of identified peaks as real $[\mathrm{M}+\mathrm{H}]^{+}$compounds and not $\left[\mathrm{M}+\mathrm{H}-\mathrm{H}_{2} \mathrm{O}\right]^{+}$nor an isotopic contribution of a main metabolite, 21 candidates were detected. Metabolites detected are described in Table 3.

\subsection{Characterization of PRED metabolites.}

PRED and metabolites M-I to M-XI were characterized by comparison with reference standards. For most of the other metabolites, tentative structures were proposed based in mass spectrometric data (Fig 1). CID mass spectra of $[\mathrm{M}+\mathrm{H}]^{+}$ion at low and moderate collision energies and of $[\mathrm{M}+\mathrm{HCOO}]^{-}$ion of metabolites $\mathrm{M}-\mathrm{XII}$ to $\mathrm{M}$-XIX are presented in Fig. 2.

Retention times and mass spectra of urinary PRED, M-I (prednisone), M-II (20ß-dihydro-

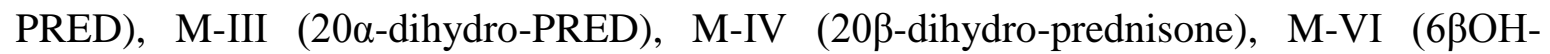
PRED) and M-XI ( $\Delta^{6}$-PRED) matched with those of commercial standards.

\section{M-V}

Metabolite $\mathrm{M}-\mathrm{V}$ was identified as $20 \alpha$-dihydro-prednisone by comparison with mass spectra of 20ß-dihydro-prednisone (Table 2, Supporting Information Fig. S1). Moreover, retention time of this metabolite matched the retention time of an impurity seen in commercial 20ß-dihydro-prednisone. 


\section{M-VII}

Metabolite M-VII was identified as $6 \alpha \mathrm{OH}-\mathrm{PRED}$ by comparison with mass spectra of 6ßOH-PRED (Table 2, Supporting Information Fig. S1). This compound was also present at low percentage $(\sim 2 \%)$ as impurity in commercial $6 \beta O H-P R E D$ standard. Retention time for $6 \alpha-\mathrm{OH}$ isomer being higher than the retention time of $6 \beta-\mathrm{OH}$ isomer is in agreement with retention times of 6-OH isomers of betamethasone ${ }^{[26]}$ and cortisol. ${ }^{[27]}$

\section{M-VIII, M-IX and M-X}

Metabolites M-VIII to M-X were identified as 20-dihydro-6OH-PRED by comparison with standards (Table 2, Supporting Information Fig. S1). 20-dihydro-6OH-PRED isomers were obtained by chemical reduction of $6 \beta O H-P R E D$ standard. With this reaction, two major peaks (2.2 and $2.6 \mathrm{~min}$ ) and a minor peak (3.9 min, only detected in SRM mode) were found. This minor peak (M-X, see below) could correspond to the reduction of $6 \alpha \mathrm{OH}-$ PRED that was present in the standard as impurity.

Taking into account that the C20 carbonyl group present in the side chains of the pregnane and 21-hydroxypregnanes series is chemically reduced preferentially to the $20 \beta$ configuration, ${ }^{[24]}$ and that $20 \alpha$ isomers elute first than $20 \beta$ isomers, ${ }^{[28]}$ peaks at 2.2 min (MIX) and $2.6 \mathrm{~min}$ (M-VIII) were suggested as $20 \alpha$ and $20 \beta$ isomers of $6 \beta, 11 \beta, 17 \alpha, 20,21$ pentahydroxypregnan-1,4-diene-3-one, respectively. In the same way, the peak at 3.9 min

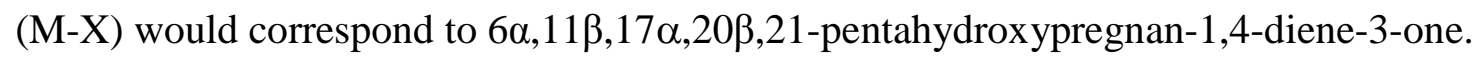

\section{M-XII and M-XIII}

Molecular mass (MM) of metabolites M-XII and M-XIII indicated a hydroxylation plus an oxidation with respect to PRED. In CID mass spectrum of $[\mathrm{M}+\mathrm{H}]^{+}$, NLs of $28(339>311)$ and 44 Da (375>331) were observed. NL of 28 Da indicates a ketone group in C11. ${ }^{[4-6,26]}$ 
NL of 44Da had been recently reported for $6 \beta$-hydroxy-triamcinolone acetonide, ${ }^{[9]}$ and $6 \beta$ hydroxy-dexamethasone, ${ }^{[26]}$ and was due to the loss of acetaldehyde, only seen after a loss of HF that originated a ketone group in C11 for both compounds. Therefore, these NL suggest a ketone group in C11.

At $30 \mathrm{eV}$, the base peak was at $\mathrm{m} / \mathrm{z} 227$ the same as in mass spectra of $6 \beta$-hydroxytriamcinolone acetonide ${ }^{[9]}$ and $6 \beta$-hydroxy-dexamethasone. ${ }^{[26]}$ After the loss of HF, these two compounds have same structure in A, B and C rings as proposed structures of metabolites M-XII and M-XIII. A plausible structure for this ion is depicted in Fig. 3A.

CID mass spectra of $\left[\mathrm{M}+\mathrm{HCOO}^{-}\right.$at $15 \mathrm{eV}$ showed a main peak at $\mathrm{m} / \mathrm{z} 343$ (NL $76 \mathrm{Da}$ ) indicating presence of a ketone in C20. There was also a NL of 28 Da (343>315), characteristic of corticosteroids with a ketone in C11 position. ${ }^{[4-6,9]}$

Thus, $6 \beta$ - and $6 \alpha-\mathrm{OH}$-prednisone were proposed for metabolites M-XII and M-XIII, respectively. Assignation of stereochemistry in C6 was based on their retention times (see M-VII section).

\section{M-XIV}

MM of metabolite M-XIV indicated a hydroxylation plus a reduction with respect to PRED. In CID mass spectra of $[\mathrm{M}+\mathrm{H}]^{+}$at low $\mathrm{CE}$, only NLs of water were seen. CID mass spectra of $\left[\mathrm{M}+\mathrm{HCOO}^{-}\right.$at $15 \mathrm{eV}$ showed a main peak at $\mathrm{m} / \mathrm{z} 347$ (NL $76 \mathrm{Da}$ ) indicating no reduction of C20 ketone. Discarding C20 reduction, the most plausible reduction would take place in $\Delta^{4}$ double bond, and therefore we suggest $6 \beta, 11 \beta, 17 \alpha, 21$-tetrahydroxy-5$\xi$ pregn-1-en-3,20-dione as plausible structure for M-XIV. Stereochemistry on C6 position was based on the much higher amounts of M-VI compared to M-VII.

\section{M-XV and M-XVI}


MM of metabolites M-XV and M-XVI suggested a hydroxylation plus two reduction steps with respect to PRED. In CID mass spectra of $[\mathrm{M}+\mathrm{H}]^{+}$at low $\mathrm{CE}$, besides NLs of water, NLs of 60 Da were seen (345>285 and 327>267) as for 20-dihydro-PRED and 20dihydro-6ßOH-PRED (Table 2) and C20 reduced metabolites of betamethasone. ${ }^{[26]}$ This fact suggested a reduction of the C20 ketone and it is in agreement with the NL of $46 \mathrm{Da}$ seen in the CID mass spectra of $\left[\mathrm{M}+\mathrm{HCOO}^{-}\right.$(Fig 4, Table 2). Besides the identified C20 reduction, the most feasible reduction step is the saturation of $\Delta^{4}$ double bond, as for metabolite M-XIV. Thus, $20 \beta$ and $20 \alpha$ isomers of $6 \beta, 11 \beta, 17 \alpha, 20,21$-pentahydroxy-5$\xi$ pregn-1-en-3-one are suggested as plausible structures for M-XV and M-XVI, respectively. The stereochemistry in C20 was assigned based on retention times of the metabolites.

\section{M-XVII}

MM of M-XVII suggested two oxidations with respect to PRED. In CID mass spectra of $[\mathrm{M}+\mathrm{H}]^{+}$at low CE, NLs of $30(339>309)$ and 26 Da (321>295) were observed (Fig 2.E).

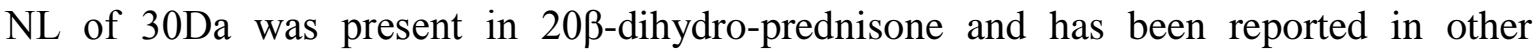
corticosteroids, ${ }^{[5,6]}$ but its origin is not clear. The NL of 26 Da corresponds to a loss of acetylene. ${ }^{[2]}$ This loss could be explained by the mechanism proposed in Fig. 3B. Similar mechanisms had been reported to explain the fragmentation of other steroids. ${ }^{[29,30]}$ This alternative fragmentation pathway could explain the absence of a NL of 28Da, typical of steroids with a ketone in C11. ${ }^{[4-6,9,26]}$ At high CE, the base peak at $m / z 171$ suggested a double bond in C6.(Table 2, Supporting Information Fig. S1)

CID mass spectra of $[\mathrm{M}+\mathrm{HCOO}]^{-}$at $15 \mathrm{eV}$ showed two main product ions, one at $\mathrm{m} / \mathrm{z} 325$ (NL $76 \mathrm{Da}$ ) and the other one at $\mathrm{m} / \mathrm{z} 201$. Ions at lower $\mathrm{m} / \mathrm{z}$ values are not common in mass spectra of corticosteroids under negative ionization, ${ }^{[4-6,9]}$ thus highly stable product ions 
were expected. Ion at $\mathrm{m} / \mathrm{z} 201$ could be explained by a retro-Diels-Alder reaction of the ion $\left[\mathrm{M}+\mathrm{HCOO}^{-}\right.$(Fig. 3C).

Taking into account all this information, 17 ,21-dihydroxy-pregnan-1,4,6-trien-3,11,20trione is suggested as a plausible structure for M-XVII.

\section{M-XVIII}

MM of M-XVIII indicated a reduction with respect to PRED. In CID mass spectrum of $[\mathrm{M}+\mathrm{H}]^{+}$at low CE, besides NLs of water, NLs of $30 \mathrm{Da}(327>297)$ and $60 \mathrm{Da}(327>267)$ (Fig 2.F) were seen, as in mass spectra of 20ß-dihydro-prednisone (Table 2, Supporting Information Fig. S1). At high CE,a main ion at $m / z 69$ was detected. This product ion has been reported in mass spectra of steroids with 1-ene-3-keto moiety and its abundance was

higher in mass spectra of the $5 \alpha$-isomer compared to the $5 \beta .^{[31,32]}$ CID mass spectra of $\left[\mathrm{M}+\mathrm{HCOO}^{-}\right.$at $15 \mathrm{eV}$ showed a main peak at $\mathrm{m} / \mathrm{z} 361$ (NL $\left.46 \mathrm{Da}\right)$ indicating no ketone group in C20.

A plausible structure for this metabolite is $17 \alpha, 20 \xi, 21$-trihydroxy-5 $\xi$-pregn-1-en-3,11dione. CID mass spectrum of $[\mathrm{M}+\mathrm{H}]^{+}$of $\mathrm{M}-\mathrm{XVIII}$ at $10 \mathrm{eV}$ (Fig.2) showed the same ions (at $\mathrm{m} / \mathrm{z}$ 363, 345, 327, 309, 297, 285 and 267) as those reported in the product ion mass spectra of 5,20-tetrahydroprednisones isomers. ${ }^{[18]}$ Both 5-epimers were mentioned in that work with the same retention time, but no attempt to resolve them was mentioned, so it is not clear if both of them were present. ${ }^{[18]}$

\section{M-XIX}

MM of M-XIX suggested a reduction of PRED. In the CID mass spectrum of $[\mathrm{M}+\mathrm{H}]^{+}$at low CE, only NLs of water were seen. The NL of 46 Da seen in CID mass spectrum of $[\mathrm{M}+\mathrm{HCOO}]^{-}$suggested a reduction of the C20 ketone group. Both 20-dihydro-PRED 
isomers had already been identified (M-II and M-III), and, for that reason, an additional reduction and an additional oxidation are expected for M-XIX.

Kraiem et $\mathrm{al}^{[17]}$ reported two isomers with similar mass spectra in positive mode as MXIX, and they suggested them as $3 \alpha$ and $3 \beta, 5$-tetrahydroprednisone. However, these structures are not in agreement with the NL of 46 Da (20-dihydro) detected in negative ionization mode for M-XIX. Additional information is needed to propose a feasible structure for M-XIX.

\section{M-XX}

MM of M-XX indicated a hydroxylation plus a reduction with respect to PRED. This compound was not detected under positive ionization, suggesting a reduction of the ketone group of the A ring. NL of 76 Da under negative ionization suggested that C20 ketone remains intact. More information is needed to propose a reliable structure for this metabolite.

\section{Other aspects}

In the biosynthesis of 1-pregnene-3-oxo metabolites (M-XIV, M-XV, M-XVI and MXVIII), after the reduction of $\Delta^{4}$ double bond isomers $5 \alpha$ and $5 \beta$ could be formed. Not all potential metabolites were detected. Different plausible explanations could be drawn: only one of the isomers was formed, the concentrations of some isomers were below our limit of detection or the isomers were not resolved in the chromatographic conditions used. Stereochemistry of the detected metabolites could not be established, except for M-XIX, because the base peak at $\mathrm{m} / \mathrm{z} 69$ in the mass spectrum of $[\mathrm{M}+\mathrm{H}]^{+}$at $30 \mathrm{eV}$ seems to indicate a $5 \alpha$ configuration. ${ }^{[31,32]}$ Endogenous corticosteroids are reduced preferentially to 
the $5 \beta$ configuration, ${ }^{[33]}$ however, it has to be taken into account that the extra double bond in C-1 of PRED could change the preferred configuration.

Among the ten 6-hydroxy metabolites detected in the present work, only $6 \beta$ OH-PRED was previously reported. Derivatization of these compounds is even more complicated than other PRED metabolites due to presence of extra hydroxyl group, and this fact could explain why these compounds were not detected in previous studies using GC-MS. ${ }^{[15,16]}$ These metabolites were also unreported by previous LC-MS/MS methods. ${ }^{[17,18]}$ This fact can be due to a co-elution with $6 \beta O H-P R E D$, which is circumvented by the UHPLCMS/MS method used in this present work. The low starting percentage of organic phase, the gentle slope and the inherent higher resolution of the UHPLC would allow for the chromatographic separation of isomeric hydroxyl-metabolites of PRED.

On the other hand, among all previously reported A-ring reduced metabolites, such as 4,5dihydro, ${ }^{[15,18]} 3,4,5$-tetrahydro ${ }^{[16]}$ or $1,2,4,5$-tetrahydro ${ }^{[15]}$ metabolites, only M-XVIII $(4,5$ dihydroprednisone) was found in our lab. This fact could be due to the most suitable detection of these compounds by GC-MS than LC-MS/MS. In electrospray ionization, the 3-keto group is protonated mainly when conjugated with double bonds; ${ }^{[4,34]}$ so when double bonds are reduced, the intensity of the compounds decrease. On the other hand, most of the open screening methods used are based on the mass spectrometric behavior of 3-keto containing corticosteroids failing for the proper detection of polyreduced metabolites. The development and application of more specific methods for these compounds might result in the detection of additional metabolites.

\subsection{Analysis of excretion studies}

Once all metabolites were identified, the whole set of samples collected after oral administration of PRED was analyzed. An LC-MS/MS method in SRM mode was 
developed for such a purpose (Table 3). The optimized SRM method allowed the detection of PRED at a limit of detection around $0.1 \mathrm{ng} / \mathrm{mL}$. PRED had been detected endogenously in urine at concentrations 100-times lower than cortisol (around $0.5 \mathrm{ng} / \mathrm{mL}$ ). ${ }^{[35]}$ This fact explains the small signal for PRED and most of its metabolites in pre-administration samples. The normalized area ratios of all metabolites along time are depicted in Fig. 4. In post-administration samples, PRED, prednisone (M-I), 20ß-dihydro-PRED (M-II) seem

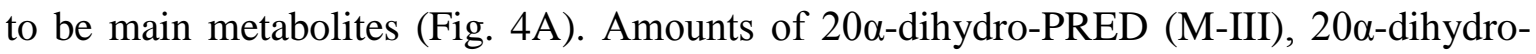
prednisone (M-V) and 20ß-dihydro-prednisone (M-IV) were also important. Thus, besides equilibrium between 11-hydroxy and 11-keto form, reduction of C20 ketone is the main biotransformation pathway in PRED metabolism. Both PRED and prednisone were hydroxylated in C6 forming the $\alpha$ and the $\beta$ isomers. Similar to the metabolism of budesonide $^{[5]}$ and triamcinolone acetonide, ${ }^{[9]}$ amounts of 6ßOH-PRED (M-VI) were much higher than those of its $6 \alpha$ isomer (M-VII) on the other hand, amounts of M-XII and M-

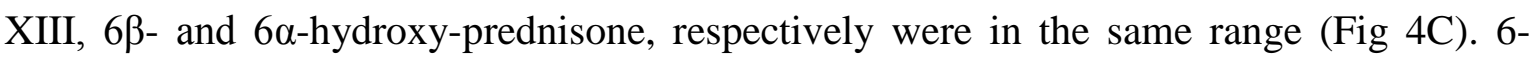
hydroxylated metabolites also suffered reduction of C20 ketone (M-VIII to M-X) and of the $\Delta^{4}$ double bond (M-XIV to M-XVI). Low abundances of metabolites M-XI and MXVII (Fig. 4B) and no detection of $16 \alpha \mathrm{OH}-\mathrm{PRED}$ seemed to indicate that $\Delta^{6}$ unsaturation and 16-hydroxylation are, respectively not important pathways in PRED metabolism. It has to be taken into account that excretion rates are not accurate because the area response is not only proportional to the concentration and also depends on the ionization and fragmentation of each compound. Moreover, the intesity of the two ISTDs was different (6ßOH-dexamethasone had in general around 3 times higher intensity than PRED- $\mathrm{d}_{8}$ ).

Some metabolites, such as PRED and M-I, showed a maximum concentration in urine in the period 0-8h after intake, and then their amounts decrease rather fast. On the other hand, other metabolites, such as M-II, M-XIV or M-XVIII, have similar amounts in the periods 
0-8h and 8-24h. Metabolite M-XIX showed maximum concentrations around 24-36h after oral intake in both volunteers.

PRED metabolic pathway is in agreement with metabolism of methylprednisolone, where the main metabolite was a 20-hydroxy-methylprednisolone. ${ }^{[6]}$ For methylprednisolone, however, intensities of $20 \alpha$-hydroxy metabolite were higher than those of the $20 \beta$-hydroxy

metabolite. ${ }^{[6]}$ In both cases, oxidation of C11 hydroxyl group is an important step in their metabolism.

C20 reduction was less important in the metabolism of triamcinolone acetonide ${ }^{[9,10]}$ and budesonide. $^{[5,7]}$ For these corticosteroids hydroxylation play a higher role in their metabolism. This different metabolism was probably due to a presence of a ketal moiety, that made these compounds more hydrophobic than PRED and methylprednisolone. Thus, a modification to biosynthesize a more hydrophilic compound was favoured.

\section{CONCLUSIONS}

Metabolism of PRED was studied using LC-MS/MS technology. With the use of open scan (precursor ion and neutral loss) methods PRED and twenty metabolites were detected in post-administration urine samples. Parent compound and metabolites M-I to M-XI were characterized by comparison with standards. A study of mass spectrometry fragmentation of PRED, its commercially available metabolites and other corticosteroids gave us important structural information of product ions. This knowledge helped us to propose reliable structures for seven of the other nine detected metabolites. Among those seven, only M-XVIII has been previously reported as a PRED metabolite. ${ }^{[18]}$

Metabolism of PRED consisted mainly in oxidation of hydroxyl group in C11 (M-I), reduction of ketone in C20 (M-II and M-III) and 6-hydroxylation (M-VI and M-VII), or a combination of some of them (M-IV and M-V, M-VIII to M-X and M-XII and M-XIII). 
Biosynthesis of metabolites M-XIV, M-XV, M-XVI and M-XVIII involved a reduction of $\Delta^{4}$ double bond combined with one of the main biotransformations mentioned above. Finally, $\Delta^{6}$ unsaturation seems to be a minor metabolic pathway, amounts of metabolites M-XI and M-XVII were almost negligible. However, it has to be taken into account that this study is designed for the detection of the main metabolites (excreted either unconjugated or conjugated with glucuronic acid) whereas $\Delta^{6}$ steroid metabolites are reported to be preferably conjugated with cysteine. ${ }^{[36]}$

The deeper understanding of the metabolism of PRED here afforded may be useful to investigate differences on the metabolites excreted in urine depending of the route of administration.

\section{Acknowledgements}

Grant from Ministerio de Ciencia e Innovación (Spain) (DEP2009-11454), Generalitat de Catalunya (Consell Català de l'Esport and DIUE 2014 SGR 692) and support by ISCIII contrato de formación en investigación Río Hortega (CM12/00085) are gratefully acknowledged. The authors would like to thank Magí Farré, Esther Menoyo, Marta Pérez, Soraya Martín, Clara Gibert, Núria Renau, Noemí Haro and Sandra Nacarino for their collaboration in the excretion studies and management of samples. 


\section{References}

[1] The World Anti-Doping Code. The 2014 Prohibited List. International Standard ^[World Anti-Doping Agency Web site]. Available at: https://wada-mainprod.s3.amazonaws.com/resources/files/WADA-prohibited-list-2014-EN.pdf. Accessed August 25, 2014.

[2] WADA Technical Document - TD2014MRPL ^[World Anti-Doping Agency Web site]. Available at: https://wada-mainprod.s3.amazonaws.com/resources/files/WADA-TD2014MRPL-v1-MinimumRequired-Performance-Levels-EN.pdf. Accessed August 25, 2014.

[3] R. Ventura, J. Segura, R. Berges, K. D. Fitch, A. R. Morton, S. Berruezo, C. Jimenez. Distinction of inhaled and oral salbutamol by urine analysis using conventional screening procedures for doping control. Ther. Drug Monit. 2000, 22, 277.

[4] O. J. Pozo, R. Ventura, N. Monfort, J. Segura, F. T. Delbeke. Evaluation of different scan methods for the urinary detection of corticosteroid metabolites by liquid chromatography tandem mass spectrometry. J. Mass Spectrom. 2009, 44, 929.

[5] X. Matabosch, O. J. Pozo, C. Pérez-Mañá, M. Farre, J. Marcos, J. Segura, R. Ventura. Identification of budesonide metabolites in human urine after oral administration. Anal. Bioanal. Chem. 2012, 404, 325.

[6] O. J. Pozo, J. Marcos, X. Matabosch, R. Ventura, J. Segura. Using complementary mass spectrometric approaches for the determination of methylprednisolone metabolites in human urine. Rapid Commun. Mass Spectrom. 2012, 26, 541. 
[7] X. Matabosch, O. J. Pozo, C. Pérez-Mañá, M. Farre, J. Marcos, J. Segura, R. Ventura. Discrimination of prohibited oral use from authorized inhaled treatment of budesonide in sports. Ther. Drug Monit. 2013, 35, 118.

[8] X. Matabosch, O. J. Pozo, N. Monfort, C. Pérez-Mañá, M. Farre, J. Marcos, J. Segura, R. Ventura. Urinary profile of methylprednisolone metabolites after oral and topical administrations. J. Steroid Biochem. Mol. Biol. 2013, 138, 214.

[9] X. Matabosch, O. J. Pozo, Papaseit.E., M. Farre, J. Marcos, J. Segura, R. Ventura. Detection and characterization of triamcinolone acetonide metabolites in human urine by LC-MS/MS after intramuscular administration. Rapid Commun. Mass Spectrom. 2014, 28, 1829.

[10] X. Matabosch, O. J. Pozo, C. Pérez-Mañá, Papaseit.E., J. Marcos, J. Segura, R. Ventura. Evaluation of the reporting level to detect triamcinolone acetonide misuse in sports. J. Steroid Biochem. Mol. Biol. 2015, 145, 94.

[11] J. J. Bunim, M. M. Pechet, A. J. Bollet. Studies on metacortandralone and metacortandracin in rheumatoid arthritis; antirheumatic potency, metabolic effects, and hormonal properties. J. Am. Med. Assoc. 1955, 157, 311.

[12] J. H. King, J. R. Weimer. Prednisone (meticorten) and prednisolone (meticortelone) in ophthamology; experimental and clinical studies. AMA. Arch. Ophthalmol. 1955, $54,46$.

[13] V. Garg, W. J. Jusko. Bioavailability and reversible metabolism of prednisone and prednisolone in man. Biopharm. Drug Dispos. 1994, 15, 163.

[14] A. Vermeulen, E. Caspi. The metabolism of prednisolone by homogenates of rat liver. J. Biol. Chem. 1958, 233, 54. 
[15] G. M. Rodchenkov, A. N. Vedenin, V. P. Uralets, V. A. Semenov. Characterization of prednisone, prednisolone and their metabolites by gas chromatography-mass spectrometry. J. Chromatogr. 1991, 565, 45.

[16] W. K. Jerjes, A. J. Cleare, P. J. Wood, N. F. Taylor. Assessment of subtle changes in glucocorticoid negative feedback using prednisolone: comparison of salivary free cortisol and urinary cortisol metabolites as endpoints. Clin. Chim. Acta. 2006, 364, 279.

[17] Kraiem H, Ouestati F, Ben Amor SS, Fenina N, Louostati H, in: Schanzer, W, Geyer H, Gotzmann A, Mareck U. (Eds.), Recent Advances in doping analysis (16), SPORTVERLAG Strauß, Köln, 2008, pp. 379.

[18] S. Ahi, A. Beotra, S. Dubey, A. Upadhyay, S. Jain. Simultaneous identification of prednisolone and its ten metabolites in human urine by high performance liquid chromatography-tandem mass spectrometry. Drug Test. Anal. 2012, 4, 460.

[19] O. J. Pozo, J. Marcos, R. Ventura, A. Fabregat, J. Segura. Testosterone metabolism revisited: discovery of new metabolites. Anal. Bioanal. Chem. 2010, 398, 1759.

[20] C. Gomez, O. J. Pozo, H. Geyer, J. Marcos, M. Thevis, W. Schanzer, J. Segura, R. Ventura. New potential markers for the detection of boldenone misuse. J. Steroid Biochem. Mol. Biol. 2012, 132, 239.

[21] C. Gomez, O. J. Pozo, J. Marcos, J. Segura, R. Ventura. Alternative long-term markers for the detection of methyltestosterone misuse. Steroids. 2013, 78, 44.

[22] A. Panusa, G. Aldini, M. Orioli, G. Vistoli, G. Rossoni, M. Carini. A sensitive and specific precursor ion scanning approach in liquid chromatography/electrospray ionization tandem mass spectrometry to detect methylprednisolone acetate and its metabolites in rat urine. Rapid Commun. Mass Spectrom. 2010, 24, 1583. 
[23] J. Segura, R. Ventura, C. Jurado. Derivatization procedures for gas chromatographic-mass spectrometric determination of xenobiotics in biological samples, with special attention to drugs of abuse and doping agents. J. Chromatogr. B Biomed. Sci. Appl. 1998, 713, 61.

[24] C. A. Han, C. Monder. Asymmetric reduction of 20-steroidal ketone: synthesis of corticosteroid derivatives containing the 20.alpha.-ol 21-al side chain. J. Org. Chem. 1982, 47, 1580.

[25] WADA Technical Document - TD2014EAAS ^[World Anti-Doping Agency Web site]. Available at: https://www.wada-ama.org/en/resources/sciencemedicine/td2014-eaas. Accessed November 21st, 2014.

[26] X. Matabosch, O. J. Pozo, N. Monfort, Pérez-Mañá.C., M. Farre, J. Segura, R. Ventura. Detection and characterization of betamethasone metabolites in human urine by LC-MS/MS. Drug Test. Anal., (2014) doi: 10.1002/dta.1770.

[27] H. Shibasaki, S. Okamoto, R. Inoue, M. Okita, A. Yokokawa, T. Furuta. Separation and quantitative determination of 6alpha-hydroxycortisol and 6betahydroxycortisol in human urine by high-performance liquid chromatography with ultraviolet absorption detection. Anal. Bioanal. Chem. 2012, 402, 2945.

[28] J. Marcos, N. Renau, G. Casals, J. Segura, R. Ventura, O. J. Pozo. Investigation of endogenous corticosteroids profiles in human urine based on liquid chromatography tandem mass spectrometry. Anal. Chim. Acta. 2014, 812, 92.

[29] O. J. Pozo, E. P. Van, K. Deventer, et al. Detection and characterization of a new metabolite of 17alpha-methyltestosterone. Drug Metab Dispos. 2009, 37, 2153.

[30] O. J. Pozo, E. P. Van, K. Deventer, S. Grimalt, J. V. Sancho, F. Hernandez, F. T. Delbeke. Collision-induced dissociation of 3-keto anabolic steroids and related 
compounds after electrospray ionization. Considerations for structural elucidation. Rapid Commun. Mass Spectrom. 2008, 22, 4009.

[31] O. J. Pozo, P. V. Eenoo, K. Deventer, F. T. Delbeke. Detection and characterization of anabolic steroids in doping analysis by LC-MS. TrAC Trends in Analytical Chemistry. 2008, 27, 657.

[32] O. J. Pozo, C. Gomez, J. Marcos, J. Segura, R. Ventura. Detection and characterization of urinary metabolites of boldione by LC-MS/MS. Part II: Conjugates with cysteine and N-acetylcysteine. Drug Test. Anal. 2012, 4, 786.

[33] S. M. Abel, J. L. Maggs, D. J. Back, B. K. Park. Cortisol metabolism by human liver in vitro--I. Metabolite identification and inter-individual variability. J. Steroid Biochem. Mol. Biol. 1992, 43, 713.

[34] O. J. Pozo, E. P. Van, K. Deventer, F. T. Delbeke. Ionization of anabolic steroids by adduct formation in liquid chromatography electrospray mass spectrometry. $J$. Mass Spectrom. 2007, 42, 497.

[35] M. Fidani, M. C. Gamberini, G. Pompa, F. Mungiguerra, A. Casati, F. Arioli. Presence of endogenous prednisolone in human urine. Steroids. 2013, 78, 121.

[36] A. Fabregat, A. Kotronoulas, J. Marcos, J. Joglar, I. Alfonso, J. Segura, R. Ventura, O. J. Pozo. Detection, synthesis and characterization of metabolites of steroid hormones conjugated with cysteine. Steroids. 2013, 78, 327. 
Table 1: Open scan methods used for the detection of unknown prednisolone metabolites.

\begin{tabular}{ccccccc}
\hline Method & $\begin{array}{c}\text { ESI } \\
\text { mode }\end{array}$ & Scan mode & Ions/losses & $\begin{array}{c}\text { Cone } \\
\text { Voltage }(\mathrm{V})\end{array}$ & $\begin{array}{c}\text { Collision } \\
\text { Energy }(\mathrm{eV})\end{array}$ & Structure \\
\hline PI91 & + & Precursor Ion & $77 / 91 / 105$ & 15 & $60 / 55 / 50$ & $\begin{array}{c}\text { Corticosteroid-like } \\
\text { structure }\end{array}$ \\
PI121 & + & Precursor Ion & $121 / 147 / 171$ & 15 & $50 / 30 / 30$ & $\begin{array}{c}1,4 \text {-diene } \\
\text { corticosteroids }\end{array}$ \\
NL76 & - & Neutral Loss & $76 / 94$ & 20 & $20 / 25$ & $\begin{array}{c}\text { Corticosteroid } \\
\text { with C20 ketone }\end{array}$ \\
\hline
\end{tabular}


Table 2: Characteristic fragmentation of $[\mathrm{M}+\mathrm{H}]^{+}$and $[\mathrm{M}+\mathrm{HCOO}]^{-}$of PRED and related compounds under positive and negative ionization modes, respectively.

\begin{tabular}{|c|c|c|c|c|c|c|c|c|c|c|}
\hline \multirow[b]{2}{*}{ Compound } & \multicolumn{6}{|c|}{ Positive ionization } & \multicolumn{4}{|c|}{ Negative ionization } \\
\hline & {$[\mathrm{M}+\mathrm{H}]^{+}$} & $\begin{array}{l}\mathrm{NL} 18 \\
\left(\mathrm{H}_{2} \mathrm{O}\right)\end{array}$ & $\begin{array}{l}\text { NL } 28 \\
\text { (CO) }\end{array}$ & NL 30 & NL 60 & $\begin{array}{l}\text { Steroid } \\
\text { Skeleton }\end{array}$ & {$[\mathrm{M}+\mathrm{HCOO}]^{-}$} & $\begin{array}{c}\text { NL 76 } \\
\text { (HCOOH+ } \\
\text { HCHO) } \\
\end{array}$ & $\begin{array}{c}\text { NL } 46 \\
\text { (HCOOH) }\end{array}$ & $\begin{array}{l}\text { NL } 28 \\
\text { (CO) }\end{array}$ \\
\hline PRED & 361 & $\begin{array}{l}361>343,343>325 \\
325>307,307>289\end{array}$ & $307>279$ & & & $\begin{array}{c}\text { 121, } 147 \\
171\end{array}$ & 405 & $405>329^{*}$ & $405>359$ & \\
\hline prednisone & 359 & $\begin{array}{l}359>341,341>323 \\
323>305,313>295\end{array}$ & $\begin{array}{l}341>313 \\
323>295\end{array}$ & & & $\begin{array}{c}121,147 \\
171\end{array}$ & 403 & $403>327^{*}$ & $403>357$ & $327>299$ \\
\hline $\begin{array}{l}\text { 20-dihydro- } \\
\text { PRED }\end{array}$ & 363 & $\begin{array}{l}363>345,345>327 \\
327>309,309>291\end{array}$ & & & $327>267$ & $\begin{array}{c}121,147 \\
171\end{array}$ & 407 & $407>331$ & $407>361^{*}$ & \\
\hline $\begin{array}{l}\text { 20-dihydro- } \\
\text { prednisone }\end{array}$ & 361 & $\begin{array}{l}361>343,343>325 \\
325>307,315>297 \\
313>295,307>289 \\
297>279,295>277\end{array}$ & $343>315$ & $343>313$ & $325>265$ & $\begin{array}{c}121,147 \\
171\end{array}$ & 405 & $405>329$ & $405>359^{*}$ & \\
\hline $\begin{array}{l}6 \beta \mathrm{OH}- \\
\text { PRED }\end{array}$ & 377 & $\begin{array}{l}377>359,359>341 \\
341>323,323>305\end{array}$ & $305>277$ & & & $\begin{array}{c}121,147 \\
171\end{array}$ & 421 & $421>345^{*}$ & $421>375$ & \\
\hline $\begin{array}{c}\text { 20-dihydro- } \\
\text { 6ßOH- } \\
\text { PRED }\end{array}$ & 379 & $\begin{array}{l}379>361,361>343 \\
343>325,325>307\end{array}$ & & & $343>283$ & $\begin{array}{c}121,147 \\
171\end{array}$ & 423 & & $423>377^{*}$ & \\
\hline$\Delta^{6}$-PRED & 359 & $\begin{array}{c}359>341,341>323 \\
323>305\end{array}$ & $305>277$ & & & 171 & 403 & $403>327^{*}$ & $403>357$ & \\
\hline $\begin{array}{l}\text { 16aOH- } \\
\text { PRED }\end{array}$ & 377 & $\begin{array}{l}377>359,359>341 \\
341>323,323>305\end{array}$ & $\begin{array}{l}323>295 \\
305>277\end{array}$ & & & $\begin{array}{c}\text { 121, } 147 \\
171\end{array}$ & 421 & $421>345$ & $421>375$ & \\
\hline
\end{tabular}

Main product ion under negative ionization 
Table 3: Metabolites of PRED detected in urine after oral administration. Molecular mass (MM) and retention times (RT) obtained by LCMS/MS, ions corresponding to $[\mathrm{M}+\mathrm{H}]^{+}$and/or $[\mathrm{M}+\mathrm{HCOO}]^{-}$and ion transitions used in the selected reaction monitoring method (SRM) to monitor each metabolite. CV: cone voltage (V); CE: collision energy (eV)

\begin{tabular}{|c|c|c|c|c|c|c|}
\hline Metabolite & $\begin{array}{c}\mathrm{RT} \\
\text { (min) }\end{array}$ & MM & {$[\mathrm{M}+\mathrm{H}]^{+}$} & {$[\mathrm{M}+\mathrm{HCOO}]^{-}$} & Metabolic pathway & SRM Transition (mode, VC, CE) \\
\hline PRED & 13.5 & 360 & 361 & 405 & - & $361>147(\mathrm{ESI}+, 10,25)$ \\
\hline Prednisone (M-I) & 13.3 & 358 & 359 & 403 & oxidation & $359>267(\mathrm{ESI}+, 15,15)$ \\
\hline 20ßOH-PRED (M-II) & 12.2 & 362 & 363 & 407 & reduction & $363>171(\mathrm{ESI}+, 10,20)$ \\
\hline 20๙OH-PRED (M-III) & 11.4 & 362 & 363 & 407 & reduction & $363>171(\mathrm{ESI}+, 10,20)$ \\
\hline 20ßOH-prednisone (M-IV) & 11.9 & 360 & 361 & 405 & reduction + oxidation & $361>265(\mathrm{ESI}+, 10,20)$ \\
\hline $\mathrm{M}-\mathrm{V}$ & 11.5 & 360 & 361 & 405 & reduction + oxidation & $361>265(\mathrm{ESI}+, 10,20)$ \\
\hline 6ßOH-PRED (M-VI) & 3.4 & 376 & 377 & 421 & hydroxylation & $377>341(\mathrm{ESI}+, 10,5)$ \\
\hline M-VII & 4.8 & 376 & 377 & 421 & hydroxylation & $377>341(\mathrm{ESI}+, 10,5)$ \\
\hline M-VIII & 2.6 & 378 & 379 & 423 & hydroxylation + reduction & $379>265(\mathrm{ESI}+, 10,10)$ \\
\hline M-IX & 2.2 & 378 & 379 & 423 & hydroxylation + reduction & $379>265(\mathrm{ESI}+, 10,10)$ \\
\hline$M-X$ & 3.9 & 378 & 379 & 423 & hydroxylation + reduction & $379>265(\mathrm{ESI}+, 10,10)$ \\
\hline$\Delta^{6}-\mathrm{PRED}(\mathrm{M}-\mathrm{XI})$ & 13.1 & 358 & 359 & 403 & oxidation & 359>171(ESI +, 15, 25) \\
\hline M-XII & 5.4 & 374 & 375 & 419 & hydroxylation + oxidation & $375>227(\mathrm{ESI}+, 10,30)$ \\
\hline M-XIII & 6.8 & 374 & 375 & 419 & hydroxylation + oxidation & $375>227(\mathrm{ESI}+, 10,30)$ \\
\hline M-XIV & 4.4 & 378 & 379 & 423 & hydroxylation + reduction & $423>347$ (ESI -, 15, 20) \\
\hline $\mathrm{M}-\mathrm{XV}$ & 12.7 & 380 & 381 & 425 & hydroxylation +2 reductions & $381>267(\mathrm{ESI}+, 10,30)$ \\
\hline M-XVI & 11.8 & 380 & 381 & 425 & hydroxylation +2 reductions & $381>267(\mathrm{ESI}+, 10,30)$ \\
\hline M-XVII & 12.9 & 356 & 357 & 401 & 2 oxidations & $357>295(\mathrm{ESI}+, 10,12)$ \\
\hline M-XVIII & 14.7 & 362 & 363 & 407 & oxidation +2 reductions & 407>361 (ESI -, 15, 20) \\
\hline M-XIX & 13.2 & 362 & 363 & 407 & oxidation +2 reductions & 407>361 (ESI -, 15, 20) \\
\hline $\mathrm{M}-\mathrm{XX}$ & 13.7 & 378 & - & 423 & hydroxylation + reduction & $423>347$ (ESI -, 15, 20) \\
\hline 6ßOH-dexamethasone (ISTD) & 7.3 & 408 & 409 & 453 & \multicolumn{2}{|c|}{$-\quad 409>227(\mathrm{ESI}+, 10,20) / / 453>377(\mathrm{ESI}-, 15,20)$} \\
\hline PRED-d 8 (ISTD) & 13.3 & 368 & 369 & 413 & \multicolumn{2}{|c|}{$369>351(\mathrm{ESI}+, 10,10) / / 413>337(\mathrm{ESI}-, 15,20)$} \\
\hline
\end{tabular}




\section{FIGURE CAPTIONS}

Fig 1. Tentative proposal of PRED metabolism in humans after oral administration.

Fig 2. CID mass spectra of PRED metabolites obtained from $[\mathrm{M}+\mathrm{H}]^{+}$ion and from $\left[\mathrm{M}+\mathrm{HCOO}^{-}\right.$ion in positive and negative electrospray ionization modes, respectively.

Fig 3. Scheme of plausible rearrangements during mass spectrometry fragmentation. Plausible structure for product ion at m/z 227 during fragmentation of M-XII and M-XIII (A). Fragmentation of $[\mathrm{M}+\mathrm{H}]^{+}$and of $[\mathrm{M}+\mathrm{HCOO}]^{-}$of $\mathrm{M}-\mathrm{XVII}(\mathrm{B}, \mathrm{C}$, respectively).

Figure 4. Excretion profile of PRED metabolites after oral administration. Corrected ratios of the areas of the metabolites and the ISTD during the first 6 days after administration are plotted. A) PRED and metabolites M-I to M-V, B) metabolites M-XI and M-XV to M-XX and C) metabolites M-VI to M-X and M-XII to M-XIV. 


\section{Detection and characterization of prednisolone metabolites in}

\section{human urine by LC-MS/MS.}

Xavier Matabosch ${ }^{1}$, Oscar J. Pozo ${ }^{1}$, Clara Pérez-Mañá, ${ }^{2,3}$, Magí Farré2,3 ${ }^{2,3}$ Jordi Segura ${ }^{1,4}$, and Rosa Ventura ${ }^{1,4}$

${ }^{1}$ Bioanalysis Research Group, IMIM (Institut Hospital del Mar d’Investigacions Mèdiques), Doctor Aiguader 88, 08003 Barcelona, Spain.

${ }^{2}$ Human Pharmacology and Clinical Neurosciences Research Group, IMIM, Doctor Aiguader 88, 08003 Barcelona, Spain.

${ }^{3}$ Department of Pharmacology, Therapeutics and Toxicology, Universitat Autònoma de Barcelona, Bellaterra (Cerdanyola del Vallès), 08193 Barcelona, Spain.

${ }^{4}$ Department of Experimental and Health Sciences, Universitat Pompeu Fabra, Doctor Aiguader 88, 08003 Barcelona, Spain.

*Correspondence to: Rosa Ventura, Bioanalysis Research Group, IMIM, Institut Hospital del Mar d’Investigacions Mèdiques, Doctor Aiguader 88, 08003 Barcelona, Spain E-mail: rventura@imim.es 


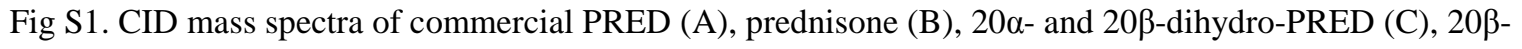

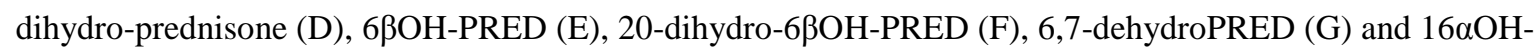
PRED in LC-MS/MS analysis obtained from $[\mathrm{M}+\mathrm{H}]^{+}$ion and from $[\mathrm{M}+\mathrm{HCOO}]^{-}$ion in positive and negative electrospray ionization modes, respectively.

MSMS $[\mathrm{M}+\mathrm{H}]^{+} 10 \mathrm{eV} \quad \mathrm{MSMS}[\mathrm{M}+\mathrm{H}]^{+} 30 \mathrm{eV} \quad \mathrm{MSMS}[\mathrm{M}+\mathrm{HCOO}]^{-}$
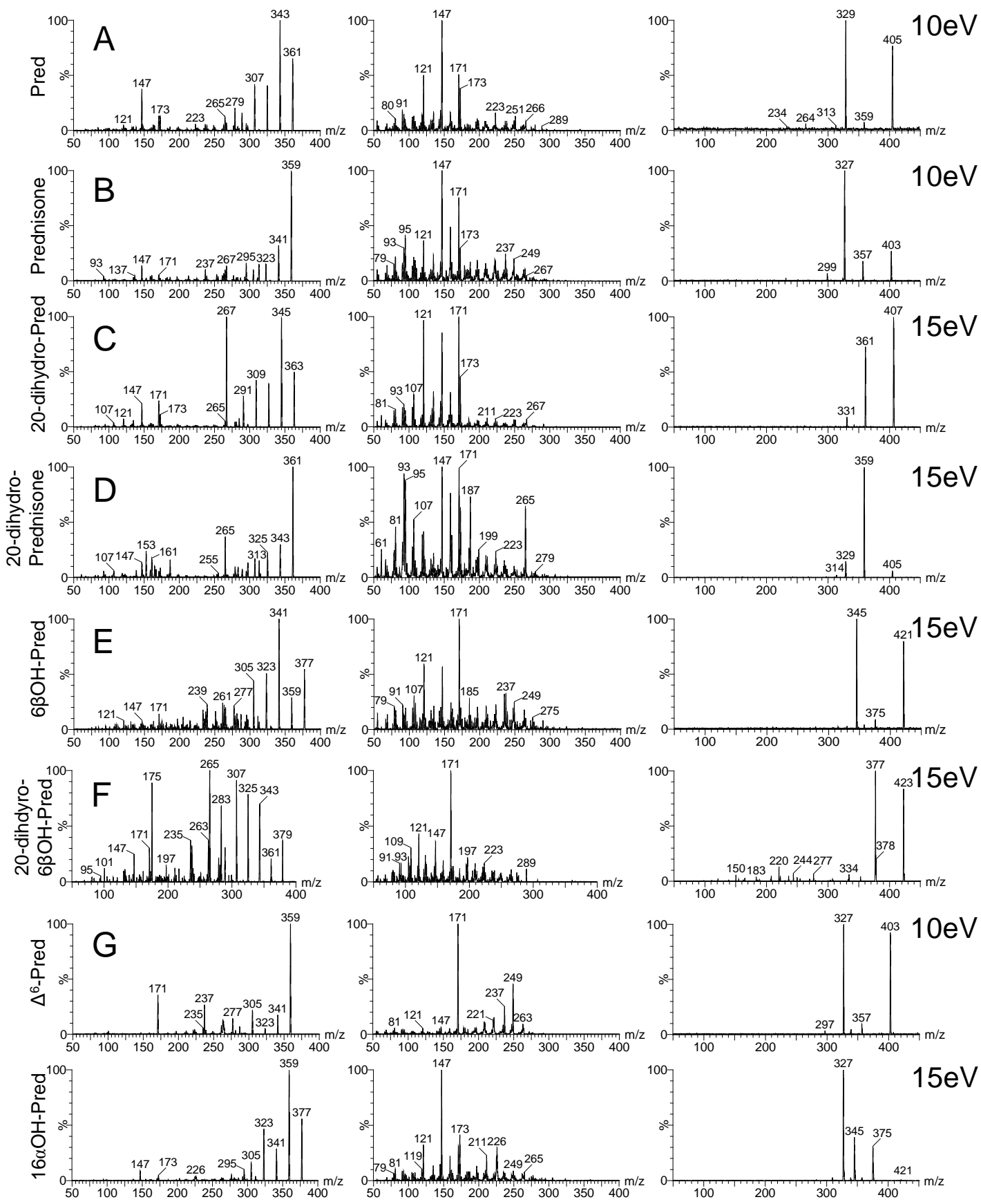

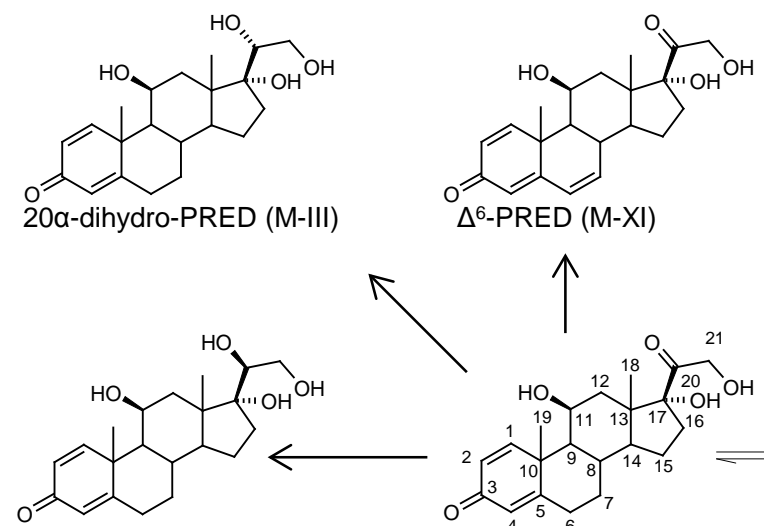

20ß-dihydro-PRED (M-II)

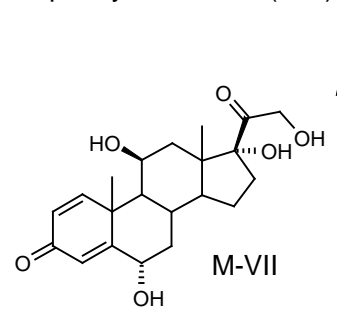

$\downarrow$<smiles>[X][M]C1CC[C@](O)([C@H](O)CO)C1(C)C[C@H](O)C1C(O)C2C1C[C@H](O)C1=CC(=O)C=CC12C</smiles>
$\underbrace{30}_{4} \underbrace{10}_{6}$

Prednisolone (PRED)<smiles>CCC</smiles>

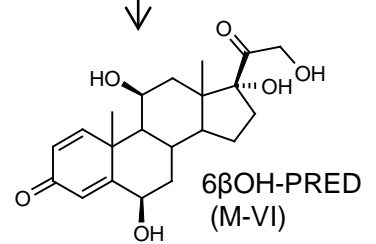

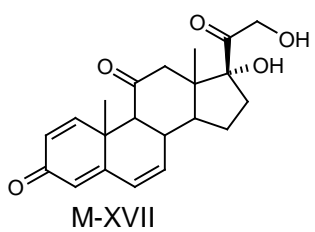

M-XVII

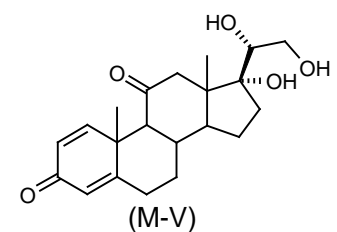

(M-V)

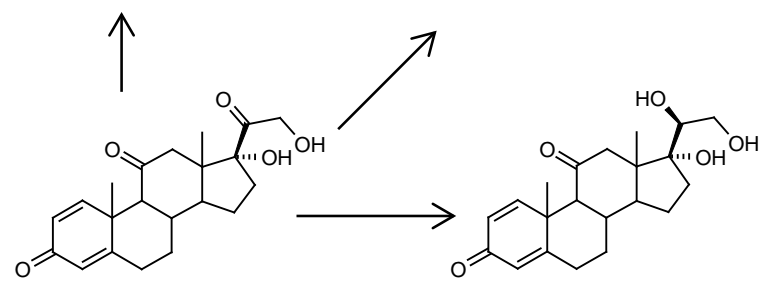

Prednisone (M-I)

20ß-dihydro-prednisone (M-IV)

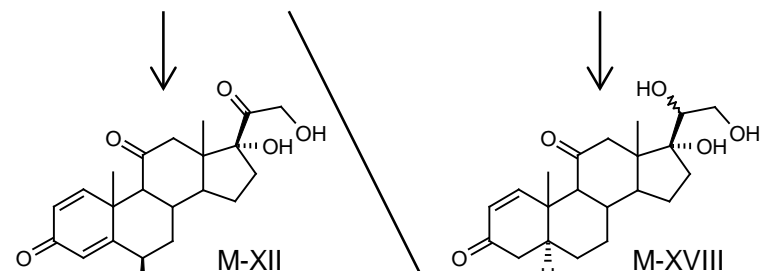

M-XII
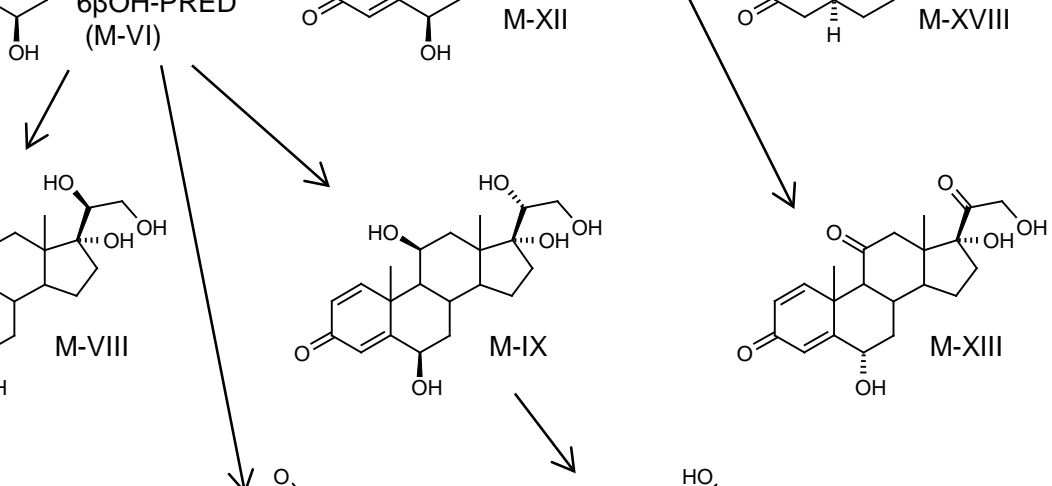<smiles>CCC(O)[C@]1(O)CCC2C3CC(O)C4CC(=O)C=CC45CC21CCC(O)C35</smiles>

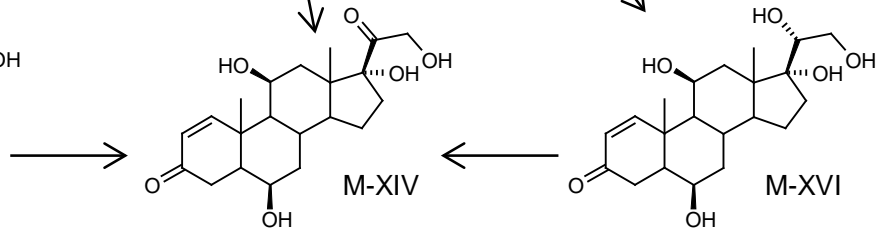




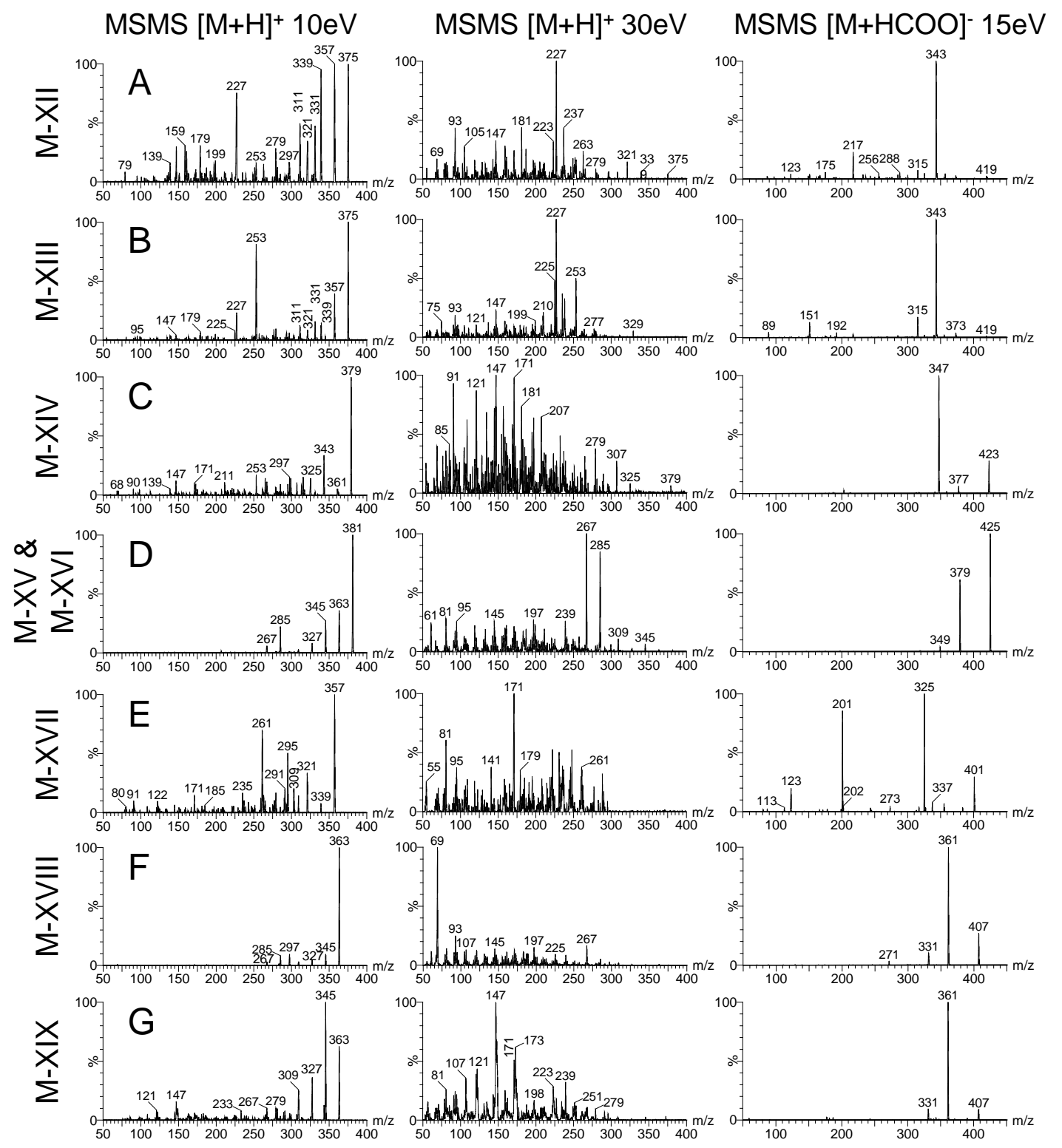




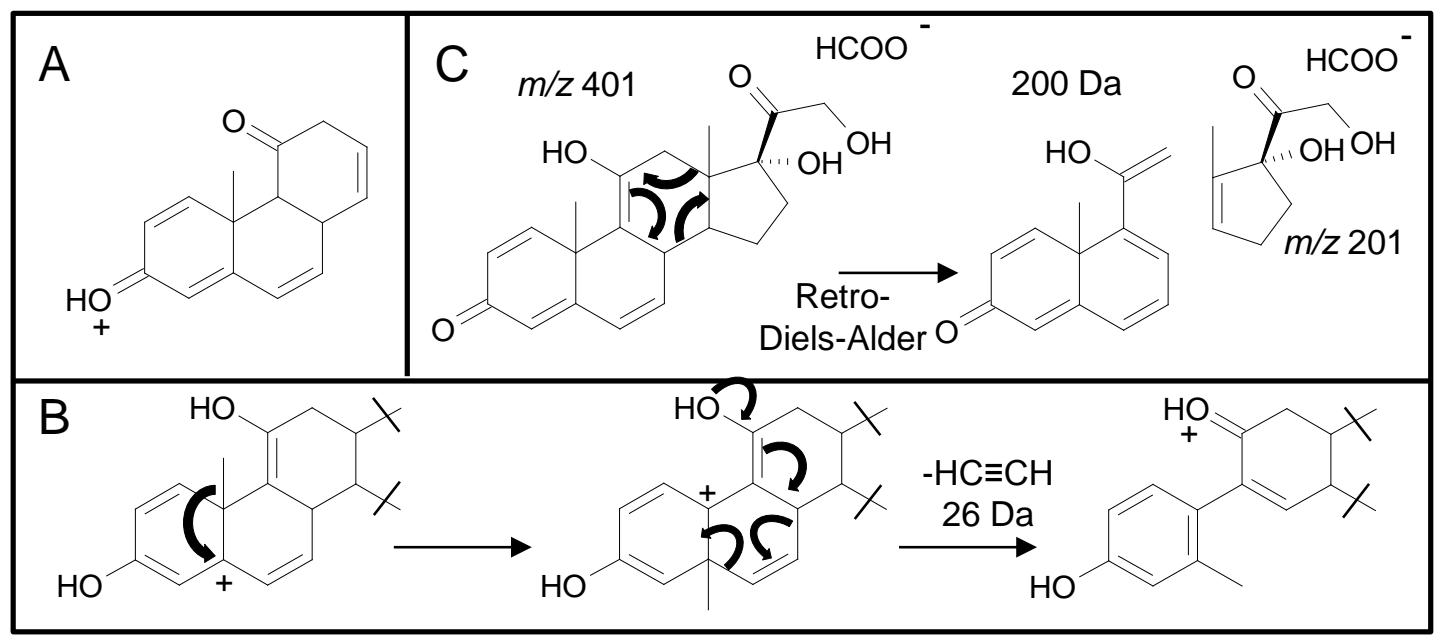




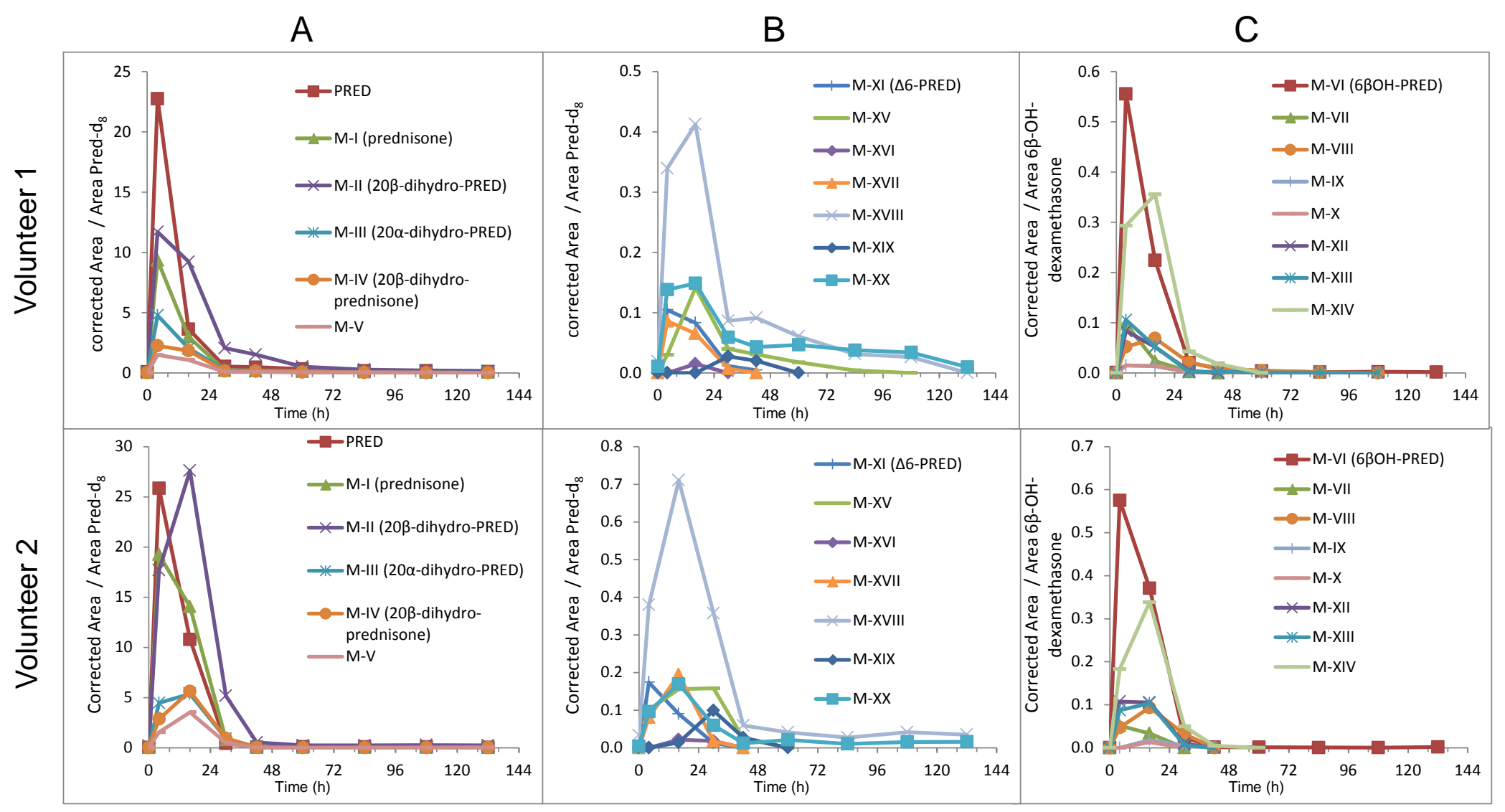

